\title{
Dirty black holes: quasinormal modes
}

\author{
A J M Medved, Damien Martin and Matt Visser \\ School of Mathematical and Computing Sciences, Victoria University of Wellington, \\ PO Box 600, Wellington, New Zealand \\ E-mail: joey.medved@mcs.vuw.ac.nz and matt.visser@mcs.vuw.ac.nz
}

Received 9 October 2003

Published DD MMM 2004

Online at stacks.iop.org/CQG/21/1 (DOI: 10.1088/0264-9381/21/0/000)

\begin{abstract}
In this paper, we investigate the asymptotic nature of the quasinormal modes for 'dirty' black holes_-generic static and spherically symmetric spacetimes for which a central black hole is surrounded by arbitrary 'matter' fields. We demonstrate that, to the leading asymptotic order, the (imaginary) spacing between modes is precisely equal to the surface gravity, independent of the specifics of the black-hole system. Our analytical method is based on locating the complex poles in the first Born approximation for the scattering amplitude. We first verify that our formalism agrees, asymptotically, with previous studies on the Schwarzschild black hole. The analysis is then generalized to more exotic black-hole geometries. We also extend considerations to spacetimes with two horizons and briefly discuss the degenerate-horizon scenario.
\end{abstract}

PACS numbers: 04.70.Dy, 03.65.Nk

\section{Introduction}

It has long been known that small perturbations of a black-hole spacetime will evolve according to a one-dimensional Schrödinger-like equation [1]. Although no (normalizable) bound-state solutions are known, there is still a very interesting class of solutions, commonly referred to as quasinormal modes [2-6], which can be used as a 'basis' for investigating the physics. The premise behind the quasinormal modes is to impose 'radiation boundary conditions' at the edges of the spacetime (typically, spatial infinity and the black-hole horizon); a constraint which necessitates a complex value for the frequency (and, hence, the 'quasi' nomenclature) [7]. As it turns out, this complex frequency can be labelled by a discrete quantum number $(n=0,1,2, \ldots)$ and takes on the following asymptotic form [8]:

$$
k_{\mathrm{qnm}}(n)=\mathrm{i} n(\mathrm{gap})+(\text { offset })+O\left[n^{-1 / 2}\right] \quad \text { as } n \rightarrow \infty,
$$

where the 'gap' and 'offset' are model-dependent, complex parameters that are determined by the precise form of the 'potential barrier' in the Schrödinger equation. 
For illustrative purposes, let us suppose an asymptotically flat spacetime in which case the gap is purely real. A useful physical interpretation is that, for a highly damped black hole (as described by asymptotically large $n$ ), the real part of the offset measures the frequency of emitted radiation, whereas the gap corresponds to quantized increments in the inverse relaxation time [7]. Hence, one might expect that both these quantities should be fundamental properties of a given black hole. In this sense, it is interesting to consider the modes for the specific case of a scalar or gravitational perturbation outside a Schwarzschild black hole (as generated numerically by Nollert [9], Andersson [10] and substantiated analytically by Motl and Neitzke [11-13]):

$$
k_{\mathrm{qnm}}(n)=\mathrm{i} \frac{1}{4 G m}\left(n+\frac{1}{2}\right)+\frac{\ln 3}{8 \pi G m}+O\left[n^{-1 / 2}\right] \quad \text { as } \quad n \rightarrow \infty,
$$

where $m$ is the black-hole mass and $G$ is Newton's constant ${ }^{1}$. For future reference, let us re-express this result directly in terms of the surface gravity at the horizon $(\kappa=1 /[4 \mathrm{Gm}]$ for a Schwarzschild black hole)

$$
k_{\mathrm{qnm}}(n)=\mathrm{i} \kappa\left(n+\frac{1}{2}\right)+\frac{\ln 3}{2 \pi} \kappa+O\left[n^{-1 / 2}\right] \quad \text { as } \quad n \rightarrow \infty .
$$

Note that the gap in the Schwarzschild case is precisely the surface gravity. One might be reasonably inclined to wonder if this occurrence is an artefact of a particularly simple model (having only one-dimensional parameter) or, rather, a resilient feature of more exotic black-hole geometries. Undoubtedly, this is an important question but, perhaps strangely, there has been limited progress towards finding the answer. Studies on quasinormal modes have, for the most part, been of a highly model-specific nature (either numerical $[9,10,14$, 15] or analytical [11-16, 17]).

In this paper, we directly address the above question with a generic analytical approach that is elegant in both its simplicity and its general applicability. (This continues a long-term project of one of the current authors - if black-hole thermodynamics is as fundamental as expected, then it should be completely independent of the detailed specifics of the black hole under consideration. The thermodynamic features of the black-hole system should be generic and unaffected by any 'dirt' that might surround the black hole [18-20].) In our analysis, we utilize the first Born approximation for the scattering amplitude [21] as a means for approximating the reflection coefficient. It is then possible to identify the quasinormal modes by locating the poles in the scattering amplitude (see, for example, [13]). Note that the Born approximation is normally thought of as a 'high-energy' approximation. More specifically, it becomes increasingly accurate as $|k| \rightarrow \infty$ (this is undoubtedly true for real $k$ and, in all likelihood, complex $k$ as well). Although this approach may appear 'quick and dirty', it does certainly confirm the known asymptotic behaviour in the Schwarzschild case. Moreover, the basic methodology can readily be extended to generic black-hole spacetimes. We ultimately show that the gap does indeed equate with the surface gravity; this being a model-independent feature of black-hole geometries.

Before proceeding, let us point out that there has been, quite recently, substantial discussion within the gravity community concerning the real part of the quasinormal frequency (i.e., the real part of the 'offset') [22-24]. This has followed, to a large degree, from Hod's observation [25] that the offset can be used to fix, uniquely, the spacing between the blackhole area eigenvalues. (This observation implies that the area of a black-hole horizon can be quantized to give an evenly spaced spectrum; a notion that was first advocated by Bekenstein

1 Here and throughout, all other fundamental constants are set to unity and a $(3+1)$-dimensional spacetime is presumed. 
[26].) Unfortunately, we are not able to address this sub-leading (offset) term at the level of the first Born approximation. Nonetheless, if quasinormal modes do indeed play some sort of role in quantum gravity (in spite of the fact that they arise out of a purely classical process), then it becomes an important matter to not only calculate but also physically motivate the complete spectral form. We would argue that the findings of this paper-specifically, identifying a universal property of quasinormal-mode spectra - can be viewed as progress in this direction.

The rest of the paper is organized in the following manner. The following section concentrates on the quasinormal modes of the Schwarzschild black hole, as this (relatively) simple case nicely illustrates the formalism and allows for a direct comparison with known results. In section 3, we go on to consider the quasinormal modes for 'generic' black-hole spacetimes (the only stipulations being staticity and spherical symmetry), including those with more than one horizon. At the end, we also discuss the conceptual difficulties of a degenerate-horizon scenario. Section 4 provides a brief summary and discussion.

\section{Schwarzschild black holes}

Although our formal techniques have quite general applicability, the focus will be, for the time being, on the minimally complicated (but nonetheless interesting) case of a Schwarzschild black hole. We are, simplistically speaking, interested in small perturbations of the spacetime outside the black-hole horizon. It is therefore appropriate to begin with a standard KleinGordon equation,

$$
\frac{1}{\sqrt{-g}} \partial_{\mu}\left[\sqrt{-g} g^{\mu \nu} \partial_{\nu}\right] \Psi(r, t, \theta, \phi)=0,
$$

where $\Psi$ is the massless perturbation field and the metric describes the background (fourdimensional Schwarzschild) spacetime. That is,

$$
\mathrm{d} s^{2}=-\left(1-\frac{2 m}{r}\right) \mathrm{d} t^{2}+\left(1-\frac{2 m}{r}\right)^{-1} \mathrm{~d} r^{2}+r^{2} \mathrm{~d} \Omega^{2} .
$$

(Note that, for the remainder, we set $G=1$. If need be, $G$ and other fundamental constants can be easily re-introduced via dimensional considerations.)

Employing a separation-of-variables technique and writing ${ }^{2}$

$$
\Psi(r, \theta, \phi, t)=\frac{1}{r} \psi(r) Y_{\ell m}(\theta, \phi) \exp (+\mathrm{i} k t),
$$

one can convert equation (4) into a Schrödinger-like equation of the form [1]

$$
\frac{\mathrm{d}^{2}}{\mathrm{~d} r_{*}^{2}} \psi-V\left[r\left(r_{*}\right)\right] \psi=-k^{2} \psi
$$

where $r_{*}$ is the so-called tortoise coordinate defined by [27]

$$
\frac{\mathrm{d} r_{*}}{\mathrm{~d} r}=\frac{1}{1-2 m / r}
$$

For scalar perturbations, the 'scattering potential' is found to be

$$
V(r)=\left(1-\frac{2 m}{r}\right)\left[\frac{\ell(\ell+1)}{r^{2}}+\frac{2 m}{r^{3}}\right],
$$

2 With the assumption that the imaginary part of $k$ is positive (cf, equation (2)) we can fix the sign in the temporal exponent by requiring the modes to be exponentially decaying in time. 
where $\ell$ is the orbital angular momentum $(l=0,1,2, \ldots)$ and let us emphasize that $V(r)$ is a rational polynomial in $r$ but not in $r_{*}$. There is a natural generalization to higher spin fields; namely [28],

$$
V(r)=\left(1-\frac{2 m}{r}\right)\left[\frac{\ell(\ell+1)}{r^{2}}+\frac{2 m\left(1-j^{2}\right)}{r^{3}}\right]
$$

and, for the physically most relevant cases,

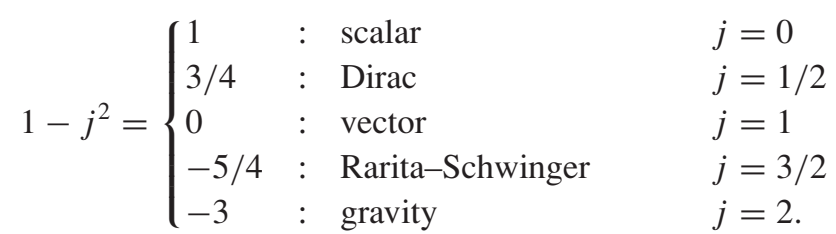

In more generic circumstances, the (generalized) tortoise coordinate will be a complicated and perhaps unsolvable function of $r$. In the present case, however, $r_{*}=r_{*}(r)$ can readily be obtained from a straightforward integration of equation (8). This process yields

$$
r_{*}(r)=r+2 m \ln \left[\frac{r-2 m}{2 m}\right] .
$$

Although this function is not explicitly invertible, we do know that the region $r \in(2 m, \infty)$ maps onto the region $r_{*} \in(-\infty,+\infty)$. That is to say, the exterior of the black hole maps onto the entire real line, and we effectively have a one-dimensional scattering problem.

It is natural, at this point, to impose the physical boundary conditions of purely outgoing plane waves at spatial infinity $\left(r_{*} \rightarrow \infty\right)$ and purely ingoing plane waves at the horizon $\left(r_{*} \rightarrow-\infty\right)$. Such boundary conditions can only be realized for complex values of $k$, thus leading to the notion of quasinormal-mode solutions to the wave equation [2]. (For the physical relevance of these modes, see the previous section; see also [7].)

For a generic (one-dimensional) scattering problem, it is known that the quasinormal modes can be identified with the poles of the reflection coefficient or, equivalently, the scattering amplitude as a function of complex asymptotic momenta [7]. Here, we will approximate the scattering amplitude by way of the first Born approximation [21]. Generally speaking, this approximation is obtained from the Fourier transform of the scattering potential with respect to the momentum transfer. More specifically, we can regard

$$
a(k) \propto \int_{-\infty}^{+\infty} V\left[r\left(r_{*}\right)\right] \exp \left[+2 \mathrm{i} k r_{*}\right] \mathrm{d} r_{*}
$$

as an approximate form of the scattering amplitude. Take note of the factor of +2 in the exponential. This follows from the fact that the momentum transfer is always minus twice the incident momentum in a one-dimensional scattering:

$$
\vec{q}=\vec{k}_{f}-\vec{k}_{i}=\left(-\vec{k}_{i}\right)-\vec{k}_{i}=-2 \vec{k}_{i}
$$

Our objective is now clear: identify the poles in equation (13) with $V(r)$ as given by equation (9). As an initial step, let us perform a change of variables,

$$
a(k) \propto \int_{2 m}^{+\infty} V[r] \exp \left[2 \mathrm{i} k r_{*}(r)\right] \frac{\mathrm{d} r_{*}}{\mathrm{~d} r} \mathrm{~d} r
$$

and then incorporate equations (8) and (12) to give

$$
a(k) \propto \int_{2 m}^{+\infty} V[r] \frac{r}{2 m} \exp [2 \mathrm{i} k r]\left[\frac{r-2 m}{2 m}\right]^{\mathrm{i} 4 m k-1} \mathrm{~d} r .
$$


A trivial shift in the integration variable then leads to

$$
a(k) \propto \int_{0}^{+\infty} V[2 m+r] \frac{2 m+r}{2 m} \exp [4 \mathrm{i} k m] \exp [2 \mathrm{i} k r]\left[\frac{r}{2 m}\right]^{\mathrm{i} 4 m k-1} \mathrm{~d} r .
$$

For future convenience, let us now define

$$
z \equiv-2 \mathrm{i} k r \quad \text { that is } \quad r=\mathrm{i} z /(2 k),
$$

and so obtain

$$
\begin{aligned}
& a(k) \propto \frac{1}{2 k}\left[\frac{\mathrm{i}}{4 m k}\right]^{\mathrm{i} 4 m k} \exp [4 \mathrm{i} k m] \\
& \quad \times \int_{0}^{+\infty} V\left[2 m+\frac{\mathrm{i} z}{2 k}\right](4 m k+\mathrm{i} z) \exp [-z] z^{\mathrm{i} 4 m k-1} \mathrm{~d} z .
\end{aligned}
$$

At this point, one can, with a brief inspection of the integrand, anticipate the presence of a linear combination of Gamma functions ${ }^{3}$. For instance, a (hypothetical) constant term in $V(z)$ would necessitate contributions that go as $\Gamma(\mathrm{i} 4 m k)$ and $\Gamma(\mathrm{i} 4 m k+1)$. The identification of poles then becomes a trivial exercise; in this case, $k_{\mathrm{qnm}}(n)=\mathrm{i} n /(4 m)$, where $n$ is any non-negative integer.

Let us now be more precise and recall our specific form (9) for the scattering potential. It follows that

$$
V\left[2 m+\frac{\mathrm{i} z}{2 k}\right]=\frac{\mathrm{i} z}{4 m k+\mathrm{i} z}\left[\frac{\ell(\ell+1)}{\left(2 m+\frac{\mathrm{i} z}{2 k}\right)^{2}}+\frac{2 m\left(1-j^{2}\right)}{\left(2 m+\frac{\mathrm{i} z}{2 k}\right)^{3}}\right] .
$$

Substituting into equation (19), we then have

$a(k) \propto \frac{\mathrm{i}}{2 k}\left[\frac{\mathrm{i}}{4 m k}\right]^{\mathrm{i} 4 m k} \exp [4 \mathrm{i} k m] \int_{0}^{+\infty}\left[\frac{\ell(\ell+1)}{\left(2 m+\mathrm{i} \frac{z}{2 k}\right)^{2}}+\frac{2 m\left(1-j^{2}\right)}{\left(2 m+\mathrm{i} \frac{z}{2 k}\right)^{3}}\right] \exp [-z] z^{\mathrm{i} 4 m k} \mathrm{~d} z$.

Since the only immediate concern is the location of the poles, it is sufficient, for our purposes, to regard $z$ as a small parameter and Taylor expand the quantity in the square brackets. Such an expansion yields a power series in ascending powers of $z$, and it soon becomes evident that we obtain

$$
a(k) \propto \sum_{s=0}^{\infty} C_{s} \Gamma(\mathrm{i} 4 m k+1+s),
$$

where each of the coefficients, $C_{s}$, is itself a regular and well-defined quantity which we do not need to calculate.

We can now readily locate the poles in the scattering amplitude. They occur at

$$
\mathrm{i} 4 m k+1=-n \quad \text { where } n \geqslant 0,
$$

so that, up to the validity of the first Born approximation (which presumably gets better as $|k|$ becomes larger), we have

$$
k_{\mathrm{qnm}}(n)=\mathrm{i} \frac{n}{4 m} \quad \text { where } \quad n>0 .
$$

Let us re-emphasize that the first Born approximation can only be expected to have validity for very large scattering energies; meaning that this result can only be trusted when $n \gg 1$ (or really when $n \rightarrow \infty)$. Fortunately, this is just the regime we are interested $\mathrm{in}^{4}$.

3 Keep in mind the primary definition of the Gamma function for positive real $n: \Gamma(n)=\int_{0}^{\infty} s^{n-1} \exp [-s] \mathrm{d} s$. Its analytic continuation to the complex plane has poles at all non-positive integers.

4 Indeed the Born series converges in relatively few cases, and is more typically an asymptotic series. Therefore, we only expect the Born approximation to be sensitive to the leading order contributions, at least when applied naively. 
Our outcome for the location of the quasinormal modes agrees, asymptotically, with the results obtained from both numerical studies [9, 10] and other analytical means [11, 12]. That is,

$$
k_{\mathrm{qnm}}(n)=\mathrm{i} \frac{n}{4 m}+O[1] \quad \text { when } n \rightarrow \infty .
$$

This spacing between asymptotic modes, or the gap, can alternatively be written as $1 / 4 m=\kappa$, where $\kappa$ is the surface gravity of the Schwarzschild black hole. At a first glance, this could be interpreted as just a happy coincidence; insofar as there is only one-dimensional parameter in the problem. (That is, the gap would almost certainly have to be the surface gravity times a numerical factor.) It will, however, be shown below that the asymptotic spacing is universally given by the relevant $\kappa$, irrespective of the details of the black-hole spacetime.

Before proceeding, let us point out that this result for the Schwarzschild gap has been known, even analytically, for quite some time. For instance, Liu and Mashoon [29], as well as Andersson [30], have made this observation by identifying the high-frequency limit of the scattering equation with the confluent hypergeometric equation. It is, however, unclear how one would translate this method, or any of the more recent analytical treatments [11, 12], into a generic setting.

\section{Generic black-hole spacetimes}

In this section, the previous formalism will be extended to 'generic' black-hole spacetimes. By generic, we mean static and spherically symmetric but with an otherwise arbitrary geometry. (So the black hole can be 'dirty' in that it may be surrounded by an arbitrary source of static and spherically symmetric matter [18-20].) Eventually, we will elaborate on spacetimes with two (or possibly more) horizons, with the second horizon not necessarily being an event horizon per se (e.g., the popular model of a Schwarzschild black hole enclosed by a de Sitter cosmological horizon, the Kottler or Schwarzschild-de Sitter geometry) ${ }^{5}$. Horizons are (for the time being) assumed to be non-degenerate; but the special case of horizon degeneracy (e.g., an extremal Reissner-Nordström black hole) will be discussed at the very end of the section.

\subsection{Single-horizon scenarios}

Given a static spacetime and spherical symmetry, the metric for a generic black hole can always be expressed, without loss of generality, in the following manner (see, for example, $[18,31])$ :

$\mathrm{d} s^{2}=-\mathrm{e}^{-2 \phi(r)}\left(1-\frac{2 m(r)}{r}\right) \mathrm{d} t^{2}+\left(1-\frac{2 m(r)}{r}\right)^{-1} \mathrm{~d} r^{2}+r^{2} \mathrm{~d} \Omega^{2}$.

Here $\phi(r)$ is a model-dependent function (related to the Morris-Thorne 'redshift function' [32]), while the 'mass' parameter $m(r)$ is equivalent to the Morris-Thorne 'shape function' [32]. Alternatively, we can write

$$
\mathrm{d} s^{2}=\mathrm{e}^{-2 \phi(r)}\left(1-\frac{2 m(r)}{r}\right)\left[-\mathrm{d} t^{2}+\frac{\mathrm{d} r^{2}}{\mathrm{e}^{-2 \phi(r)}\left(1-\frac{2 m(r)}{r}\right)^{2}}\right]+r^{2} \mathrm{~d} \Omega^{2},
$$

5 Although the formalism can also be extended to multiple-horizon scenarios, it is the feeling of the authors that, from an operative viewpoint, a single observer in any spherically symmetric geometry would be able to deduce the existence of at most two horizons. That is, for any such observer, the accessible spacetime will have no more than two spatial boundaries and, therefore, no more than two (non-degenerate) horizons can ever come into play. Hence, formal considerations will be restricted to spacetimes with one or two horizons. 
which leads, quite naturally, to a generalized tortoise coordinate,

$$
\frac{\mathrm{d} r_{*}}{\mathrm{~d} r}=\frac{1}{\mathrm{e}^{-\phi(r)}\left(1-\frac{2 m(r)}{r}\right)} ;
$$

and so

$$
\mathrm{d} s^{2}=\mathrm{e}^{-2 \phi(r)}\left(1-\frac{2 m(r)}{r}\right)\left[-\mathrm{d} t^{2}+\mathrm{d} r_{*}^{2}\right]+r^{2} \mathrm{~d} \Omega^{2} .
$$

Next, let us revisit the Klein-Gordon equation (4) for a massless perturbation field. In terms of the generic spacetime described above, this equation expands into

$$
\left\{-\partial_{t}^{2}+\frac{1}{r^{2}} \partial_{r_{*}} r^{2} \partial_{r_{*}}+\mathrm{e}^{-2 \phi(r)}\left(1-\frac{2 m(r)}{r}\right) \Delta_{2}\right\} \Psi=0
$$

where $\Delta_{2}$ represents the angular part of the d'Alembertian. We can now proceed in the standard way; namely, factoring $\Psi$ into a temporal part $[\exp (+\mathrm{i} k t)]$, angular part (the usual spherical harmonic) and a radial part. One can express the radial part as $r^{p} u\left[r\left(r_{*}\right)\right]$; in which case, the unique choice of $p$ which eliminates all terms containing $\partial_{r_{*}} u$ (but not the double derivatives) happens to be $p=-1$. (This was exactly the same exponent that occurred in the Schwarzschild case, which is now seen to be generic.) Given this choice, a Schrödinger-like form is once again obtained:

$$
\frac{\mathrm{d}^{2}}{\mathrm{~d} r_{*}^{2}} u-V\left[r\left(r_{*}\right)\right] u=-k^{2} u,
$$

except that the scattering potential is significantly more complicated than was found for the Schwarzschild case. More specifically, some straightforward calculation yields the following result: ${ }^{6}$

$$
\begin{aligned}
V(r) & =\frac{1}{r}\left(\partial_{r_{*}}^{2} r\right)+\mathrm{e}^{-2 \phi(r)}\left(1-\frac{2 m(r)}{r}\right) \frac{\ell(\ell+1)}{r^{2}} \\
& =\mathrm{e}^{-2 \phi(r)}\left(1-\frac{2 m(r)}{r}\right)\left[\frac{\ell(\ell+1)}{r^{2}}-f(r)\right],
\end{aligned}
$$

where we have defined

$$
f(r) \equiv\left(1-\frac{2 m(r)}{r}\right) \frac{\phi^{\prime}(r)}{r}+\frac{2}{r^{2}}\left(m^{\prime}(r)-\frac{m(r)}{r}\right),
$$

and a prime indicates a derivative with respect to $r$.

Provided that $f(r)$ is a well-defined and regular quantity (which must always be the case since this is equivalent to the tortoise coordinate being well behaved), it is clear that the generic potential is qualitatively very similar to that of the Schwarzschild scenario (cf equation (9)). Rather, the sticking point with the current calculation is that there is, in general, no means of obtaining a closed form solution for $r_{*}=r_{*}(r)$.

We can, however, circumvent the aforementioned difficulty by first taking note of the exact generic expression for the surface gravity [18],

$$
\kappa=\frac{1}{2 r_{h}} \mathrm{e}^{-\phi\left(r_{h}\right)}\left[1-8 \pi \rho\left(r_{h}\right) r_{h}^{2}\right]=\left.\frac{1}{2} \frac{\mathrm{d}}{\mathrm{d} r}\left[\mathrm{e}^{-\phi(r)}\left(1-\frac{2 m(r)}{r}\right)\right]\right|_{r_{h}},
$$

${ }^{6}$ Here, for the sake of simplicity, we are considering a scalar $(j=0)$ perturbation. It is, however, technically possible to extend the calculation to arbitrary $j$, and such a generalization should not alter any of our results or conclusions. 
where $r=r_{h}$ indicates the horizon (specified by $\left.2 m\left(r_{h}\right)=r_{h}\right)$ and $\rho(r)$ is the energy density. Hence we can expand equation (28) to give

$$
\begin{aligned}
\frac{\mathrm{d} r_{*}}{\mathrm{~d} r} & =\frac{1}{2 \kappa\left\{\left(r-r_{h}\right)-\alpha\left(r-r_{h}\right)^{2}+O\left[\left(r-r_{h}\right)^{3}\right]\right\}} \\
& =\frac{1}{2 \kappa\left(r-r_{h}\right)}+\frac{\alpha}{2 \kappa}+O\left[\left(r-r_{h}\right)\right],
\end{aligned}
$$

where $\alpha$ is a model-dependent constant parameter. (Although such an expansion is technically valid only in the vicinity of the horizon, it turns out to be sufficient for the purpose of identifying poles in the scattering amplitude. As seen in the previous section, the location of the poles, at the level of the first Born approximation, is only sensitive to the near-horizon geometry.) Moreover, the above expansion can now be directly integrated to yield

$$
r_{*}=\frac{\alpha}{2 \kappa}\left(r-r_{h}\right)+\frac{1}{2 \kappa} \ln \left[\frac{r-r_{h}}{r_{h}}\right]+O\left[\left(r-r_{h}\right)^{2}\right] .
$$

Beginning with the (approximate) expression for the scattering amplitude (13) and repeating the steps that took us up to equation (19), we now find that (here using the 'convenient' choice of $z \equiv-\mathrm{i} \alpha k r / \kappa$ and neglecting the irrelevant prefactors)

$a(k) \propto \int_{0}^{+\infty} V\left[r_{h}+\frac{\mathrm{i} z \kappa}{\alpha k}\right]\left(\frac{k}{\kappa}+\mathrm{i} z+O\left[z^{2}\right]\right) \exp [-z]\left(1+O\left[z^{2}\right]\right) z^{\mathrm{i}(k / \kappa)-1} \mathrm{~d} z$.

Now what about the scattering potential? An inspection of equation (33) reveals that $V\left[r_{h}+\mathrm{i} z \kappa /(\alpha k)\right]$ will translate into a power series in $z$ times several terms; each of which contains some (typically negative) power of the argument $\left[r_{h}+\mathrm{i} z \kappa /(\alpha k)\right]$. As in the previous section, we can, for our purposes, regard $z$ as small and Taylor expand appropriately. Hence, we end up with a complicated power series in $z$. Fortunately, the precise details of this expression are unimportant to us.

Given the previous expression (38) and the above discussion, it becomes clear that

$$
a(k) \propto \int_{0}^{+\infty} \exp [-z] z^{\mathrm{i} k / \kappa} F(z) \mathrm{d} z,
$$

where $F(z)$ is some unknown but, in principle, calculable power series in integer powers of $z$. (Given that the surface gravity is non-zero, the leading term in $F$ is of the order $z^{0}$, as can be seen by evaluating $\mathrm{d} V(r) / \mathrm{d} r \neq 0$ at the horizon.)

From the above form, it follows that

$$
a(k) \propto \sum_{s=0}^{\infty} C_{s} \Gamma\left(\mathrm{i} \frac{k}{\kappa}+1+s\right) .
$$

(As before, when it comes to locating the position of the poles, we do not need to calculate the coefficients $C_{s}$.) Hence, the first Born-approximated poles are located at

$$
k_{\mathrm{qnm}}(n)=\mathrm{i} n \kappa \quad \text { where } \quad n>0,
$$

which is enough to imply that the actual physical poles lie at

$$
k_{\mathrm{qnm}}(n)=\mathrm{i} n \kappa+O[1] \quad \text { where } n>0,
$$

with the constant term becoming (in comparison) irrelevant as $n \rightarrow \infty$. This is the main result of the paper and substantiates our claim at the end of section 2 . 


\subsection{Dual-horizon scenarios}

Let us now envision an observer 'trapped' between two horizons; for instance, the region of spacetime between a Schwarzschild black hole and a de Sitter cosmological horizon or the two event horizons in a Reissner-Nordström black hole (although our formalism certainly allows for much more generic situations). We propose that it is straightforward to extend the previous analysis to such situations by virtue of the following observations:

(i) Given a spherically symmetric and static spacetime, the metric can still be cast, without loss of generality, in the form of equation (26).

(ii) In the vicinity of any given horizon, the derivative $\mathrm{d} r_{*} / \mathrm{d} r$ (and all related quantities) can be expanded in the form of equation (36), where the surface gravity $(\kappa)$ and horizon location $\left(r_{h}\right)$ are uniquely defined parameters for the horizon in question.

(iii) At the level of the first Born approximation, the poles in the scattering amplitude are sensitive only to the near-horizon geometry (or geometries) of the spacetime.

To further elaborate, starting with the appropriately revised form of equation (15),

$$
a(k) \propto \int_{r_{1}}^{r_{2}} V[r] \exp \left[2 \mathrm{i} k r_{*}(r)\right] \frac{\mathrm{d} r_{*}}{\mathrm{~d} r} \mathrm{~d} r,
$$

(where $r_{1}$ and $r_{2}$ locate the two horizons in the spacetime), we can split this integral at some intermediate point ( say $r_{x}$ ) and then, by way of the third observation, make the approximation of extending the integrals to $r_{x} \rightarrow \pm \infty$. This procedure effectively yields two distinct sets of poles, one coming from each horizon. (These are actually two distinct scattering problems which can be distinguished by the orientation of the incident wave.) That is, one can anticipate the asymptotic form (with the surface gravities labelled accordingly)

$$
k_{\mathrm{qnm}}\left(n_{1}\right)=\mathrm{i} n_{1} \kappa_{1}+O[1] \quad \text { or } \quad k_{\mathrm{qnm}}\left(n_{2}\right)=\mathrm{i} n_{2} \kappa_{2}+O[1],
$$

as $n_{1 \text { or } 2} \rightarrow \infty$. Note that the inverse of the surface gravity effectively fixes the time scale, so that the modes scattered by the inner/outer horizon (i.e., the larger/smaller surface gravity) will dominate observations at earlier/later times. Further note that this phenomenon agrees with analytical estimates, based on the use of the Poschl-Teller potential, as performed by Suneeta [33].

Implicit in the above discussion is that the two horizons can indeed be spatially isolated. This immediately rules out spacetimes with degenerate (or very nearly degenerate) horizons, for instance, an extremal Reissner-Nordström black hole or a Nariai (degenerate Schwarzschild-de Sitter) spacetime. To further complicate matters, degenerate horizons invariably have a vanishing surface gravity, thus rendering our previous expansions to be useless in this context.

\subsection{Extremal horizons}

In spite of the difficulties inherent in degenerate-horizon spacetimes, one possible recourse would be to focus on the near-horizon form of the second radial derivative of $g_{t t}$ (since the first derivative vanishes at a degenerate horizon). That is to say, we can now expand $\mathrm{d} r_{*} / \mathrm{d} r$ as follows:

$$
\begin{aligned}
\frac{\mathrm{d} r_{*}}{\mathrm{~d} r} & =\left[\frac{1}{\alpha\left(r-r_{h}\right)^{2}+\beta\left(r-r_{h}\right)^{3}+\gamma\left(r-r_{h}\right)^{4}+O\left[\left(r-r_{h}\right)^{5}\right]}\right] \\
& =\frac{1}{\alpha\left(r-r_{h}\right)^{2}}-\frac{\beta}{\alpha^{2}\left(r-r_{h}\right)}+\frac{\beta^{2}-\gamma \alpha}{\alpha^{3}}+O\left[\left(r-r_{h}\right)\right],
\end{aligned}
$$


where $\alpha$ (related to the second derivative), $\beta$ and $\gamma$ are model-dependent constants. The tortoise coordinate is then

$$
r_{*}=\frac{-1}{\alpha\left(r-r_{h}\right)}-\frac{\beta}{\alpha^{2}} \ln \left[\frac{r-r_{h}}{r_{h}}\right]+\frac{\beta^{2}-\gamma \alpha}{\alpha^{3}}\left(r-r_{h}\right)+O\left[\left(r-r_{h}\right)^{2}\right] .
$$

Now, closely following the previous methodology, we find that (after shifting the $r$ integration from $\left(r_{h}, \infty\right)$ to $\left.(0, \infty)\right)$ the scattering amplitude takes on the form

$$
\begin{gathered}
a(k) \propto \int_{0}^{+\infty} V\left[r_{h}+r\right] \exp \left[-\frac{2 \mathrm{i} k}{\alpha} \frac{1}{r}+\frac{\beta^{2}-\gamma \alpha}{\alpha^{3}} 2 \mathrm{i} k r+O\left[r^{2}\right]\right] \\
\times\left(\frac{r}{r_{h}}\right)^{-2 \mathrm{i} k \beta / \alpha^{2}}\left(\frac{1}{r^{2}}-\frac{\beta}{\alpha} \frac{1}{r}+O[1]\right) \mathrm{d} r
\end{gathered}
$$

With the definition $z \equiv r / r_{h}$, the above can be rearranged into

$a(k) \propto \int_{0}^{+\infty} \exp \left[-\frac{2 \mathrm{i} k}{\alpha r_{h}} \frac{1}{z}+\frac{\beta^{2}-\gamma \alpha}{\alpha^{3}} 2 \mathrm{i} k r_{h} z\right] z^{-2 \mathrm{i} k \beta / \alpha^{2}} F(z) \mathrm{d} z$,

where $F(z)$ is again some (in principle) calculable power series in $z$ with integer exponents.

An evaluation of the $z$ integral now yields modified Bessel functions which, apart from the possibility of a trivial pole at $k=0$, do not have poles at finite $|k|$. We can physically interpret this finding as follows: when the surface gravity is non-zero, the poles in complex $k$-space can ultimately be traced back to the small- $z$ (or near-horizon) behaviour of the integrand. Whereas, in the present case of a degenerate horizon, the integrand factor $\exp (-\mathrm{i} k / r)$ washes out any possibility of a pole for $k \neq 0$.

Which is to say, by setting $\kappa \rightarrow 0$ in the general analysis, we are able to 'predict' that the quasinormal modes all collapse to zero momentum. But what this really means is that the quasinormal modes (if any exist) do not lie in a region of the complex $k$ plane where the first Born approximation is trustworthy. Thus, what we really expect for extremal black holes is that the quasinormal modes (if any exist) lie in some bounded region of the complex $k$ plane and are either finite in number or densely scattered in some bounded region.

Before concluding, let us point out some discrepancies between our analysis and other recent works that have considered the quasinormal modes of a charged black hole; both analytically [13,16] and numerically [14]. Firstly, there is evidence that the gap of a ReissnerNordström black hole - if it is periodic at all—goes, not as the surface gravity at the outer horizon, but rather as a complicated function of both surface gravities (inner and outer horizon). Naively, this is not what one would expect from our findings, given that the interior horizon is not explicitly part of the scattering problem. Secondly, the same papers have found that the highly damped modes of an extremal Reissner-Nordström black hole are formally identical to a Schwarzschild black hole of the same mass. This is clearly in contrast to our arguments above. It is quite possible that our methodology breaks down, in some subtle way, in spacetimes with a 'hidden horizon'; that is, it is feasible that the first Born approximation is unable to properly account for these added complexities. It should be noted, however, that some of these authors have also commented on interpretative difficulties [16] and numerical instabilities [14] on account of some 'peculiar features' [34] of the quasinormal spectra of charged black holes. Moreover, the Schwarzschild limit of their Reissner-Nordström spectrum does not appear to give back the desired result. We would suggest that the quasinormal-mode problem for this charged model requires further attention. 


\section{Conclusion}

To summarize, we have used a simple analytical method-based on the first Born approximation for the scattering amplitude - to locate the quasinormal modes for a static, spherically symmetric but otherwise generic black-hole spacetime. For the very special case of a Schwarzschild black hole, our methodology was found to agree, asymptotically, with the results obtained from various numerical and analytical studies. Moreover, that the asymptotic spacing or 'gap' is equivalent to the surface gravity (as is accepted in the Schwarzschild case) was shown to be a model-independent feature of a wide range of black-hole spacetimes. We also generalized this outcome to spacetimes with two horizons and reported some limited progress in the context of degenerate-horizon geometries.

The virtues of our formal treatment include generality and a straightforward analytical approach that does not obscure the physical process being investigated. Nevertheless, the simplicity of our method has a price: we have (so far) only been able to locate the leading term in the asymptotic expansion of the quasinormal mode. Meanwhile, the next-order term - that is, the 'offset' - has sparked considerable recent interest because of a conjectural relationship with the black-hole area spectrum. It is unfortunate that the Born approximation seems to diverge at higher orders, so that our approach would have to be significantly modified to reproduce the entire spectrum.

\section{Acknowledgments}

Research is supported by the Marsden Fund administered by the New Zealand Royal Society and by the University Research Fund of Victoria University.

\section{References}

[1] Regge T and Wheeler J A 1957 Stability of a Schwarzschild singularity Phys. Rev. 1081063

[2] Press W H 1971 Long wave trains of gravitational waves from a vibrating black hole Astrophys. J. 170 L105

[3] Chandrasekhar S and Detweiler S 1975 The quasi-normal modes of the Schwarzschild black hole Proc. R. Soc. Lond. A 344444

Chandrasekhar S and Detweiler S 1977 On the reflection and transmission of neutrino waves by a Kerr black hole Proc. R. Soc. Lond. A 352325

[4] Leaver E W 1985 An analytic representation for the quasi normal modes of Kerr black holes Proc. R. Soc. Lond. A 402285

[5] Chandrasekhar S 1983 The Mathematical Theory of Black Holes (Oxford: Oxford Science Publications)

[6] Andersson N and Jensen B P 2000 Scattering by black holes Preprint gr-qc/0011025

[7] See, for comprehensive reviews, Nollert H-P 1999 Quasinormal modes: the characteristic sound of black holes and neutron stars Class. Quantum Grav. 16 R159

Kokkotas K D and Schmidt B G 1999 Quasi-Normal modes of black holes and stars Living Rev. Rel. 22 (Preprint gr-qc/9909058)

[8] Bachelot A and Motet-Bachelot A 1992 Resonances of Schwarzschild black holes Proc. IV Int. Conf. of Hyperbolic Problems (Taosmina, 1992) (Braunschweig: Vieweg)

[9] Nollert H-P 1993 Quasinormal modes of Schwarzschild black holes: the determination of quasinormal frequencies with very large imaginary parts Phys. Rev. D 475253

[10] Andersson N 1993 On the asymptotic distribution of quasinormal-mode frequencies for Schwarzschild black holes Class. Quantum Grav. 10 L61

[11] Motl L 2003 An analytical computation of asymptotic Schwarzschild quasinormal frequencies Adv. Theor. Math. Phys. 61135 (Preprint gr-qc/0212096)

[12] Motl L and Neitzke A 2003 Asymptotic black hole quasinormal frequencies Adv. Theor. Math. Phys. 7307 (Preprint hep-th/0301173)

[13] Neitzke A 2003 Greybody factors at large imaginary frequencies Preprint hep-th/0304080 
[14] Berti E and Kokkotas K D 2003 Asymptotic quasinormal modes of Reissner-Nordström and Kerr black holes Phys. Rev. D 68044027 (Preprint hep-th/0303029)

[15] See, for other recent examples, Glampedakis K and Andersson N 2003 Quick and dirty methods for studying black-hole resonances Class. Quantum Grav. 203441 (Preprint gr-qc/0304030)

Xue L-H, Shen Z-X, Wang B and Su R-K 2003 Numerical simulation of quasi-normal modes in time-dependent background Preprint gr-qc/0304109

Cardoso V, Konoplya R and Lemos J P S 2003 Quasinormal frequencies of Schwarzschild black holes in anti-de Sitter spacetimes: a complete study on asymptotic behavior Phys. Rev. D 68044024 (Preprint gr-qc/0305037)

Berti E, Cardoso V, Kokkotas K and Onozawa H 2003 Highly damped quasinormal modes of Kerr black holes Preprint hep-th/0307013

Cardoso V, Lemos J P S and Yoshida S 2003 Quasinormal modes of Schwarzschild black holes in four and higher dimensions Preprint gr-qc/0309112

[16] Andersson N and Howls C J 2003 The asymptotic quasinormal mode spectrum of non-rotating black holes Preprint gr-qc/0307020

[17] See, for other recent examples, Cardoso V, Dias O J C and Lemos J P S 2003 Gravitational radiation in D-dimensional spacetimes Phys. Rev. D 67064026 (Preprint hep-th/0212168)

Cardoso V and Lemos J P S 2003 Quasinormal modes of the near extremal Schwarzschild-de Sitter black hole Phys. Rev. D 67084020 (Preprint gr-qc/0301078)

Konoplya R A 2003 Quasinormal behavior of the D-dimensional Schwarzschild black hole and higher order WKB approach Phys. Rev. D 68024018 (Preprint gr-qc/0303052)

Konoplya R A 2003 Gravitational quasinormal radiation of higher-dimensional black holes Preprint hepth/0309030

Cho H-T 2003 Dirac quasi-normal modes in Schwarzschild black hole spacetimes Phys. Rev. D 68024003 (Preprint gr-qc/0303078)

Maassen van den Brink A 2003 The WKB analysis of the Regge-Wheeler equation down in the frequency plane Preprint gr-qc/0303095

Maassen van den Brink A 2003 Approach to the extremal limit of the Schwarzschild-de Sitter black hole Phys. Rev. D 68047501 (Preprint gr-qc/0304092)

Molina C 2003 Quasinormal modes of $d$-dimensional spherical black holes with a near extreme cosmological constant Phys. Rev. D 68064007 (Preprint gr-qc/0304053)

Birmingham D 2003 Asymptotic quasinormal frequencies of $d$-dimensional Schwarzschild black holes Phys. Lett. B 569199 (Preprint hep-th/0306004)

Zhidenko A 2003 Quasi-normal modes of Schwarzschild-de Sitter black holes Preprint gr-qc/0307012

Musiri S and Siopsis G 2003 Perturbative calculation of quasi-normal modes of Schwarzschild black holes Preprint hep-th/0308168

Musiri S and Siopsis G 2003 On quasi-normal modes of Kerr black holes Preprint hep-th/0309227

Castello-Branco K H C and Abdalla E 2003 Analytic determination of the asymptotic quasi-normal mode spectrum of Schwarzschild-de Sitter black holes Preprint gr-qc/0309090

Berti E, Cavaglia M and Gualtieri L 2003 Gravitational energy loss in high energy particle collisions: ultrarelativistic plunge into a multidimensional black hole Preprint hep-th/0309203

[18] Visser M 1992 Dirty black holes: thermodynamics and horizon structure Phys. Rev. D 462445 (Preprint hep-th/9203057)

[19] Visser M 1993 Dirty black holes: entropy versus area Phys. Rev. D 48583 (Preprint hep-th/9303029)

[20] Visser M 1993 Dirty black holes: entropy as a surface term Phys. Rev. D 485697 (Preprint hep-th/9307194)

[21] See, for example, Shankar R 1980 Principles of Quantum Mechanics (New York: Plenum)

[22] Dreyer O 2003 Quasinormal modes, the area spectrum, and black hole entropy Phys. Rev. Lett. 90081301 (Preprint gr-qc/0211076)

[23] Kunstatter G $2003 d$-dimensional black hole entropy spectrum from quasi-normal modes Phys. Rev. Lett. 90 161301 (Preprint gr-qc/0212014)

[24] Also see, for instance, Corichi A 2003 On quasinormal modes, black hole entropy, and quantum geometry Phys. Rev. D 67087502 (Preprint gr-qc/0212126)

Hod S 2003 Kerr black hole quasinormal frequencies Phys. Rev. D 67081501 (Preprint gr-qc/0301122)

Hod S 2003 Asymptotic quasinormal mode spectrum of rotating black holes Preprint gr-qc/0307060

Kaul R K and Rama S K 2003 Black hole entropy from spin one punctures Phys. Rev. D 68024001 (Preprint gr-qc/0301128)

Abdalla E, Castello-Branco K H C and Lima-Santos A 2003 Area quantization in quasi-extreme black holes Mod. Phys. Lett. A 181435 (Preprint gr-qc/0301130)

Polychronakos A P 2003 Area spectrum and quasinormal modes of black holes Preprint hep-th/0304135 
Birmingham D, Carlip S and Chen Y 2003 Quasinormal modes and black hole quantum mechanics in 2+1 dimensions Class. Quantum Grav. 20 L239 (Preprint hep-th/0305113)

Swain J 2003 The Pauli exclusion principle and SU(2) vs. SO (3) in loop quantum gravity Preprint gr-qc/0303073 Oppenheim J 2003 The spectrum of quantum black holes and quasinormal modes Preprint gr-qc/0307089

Ling Y and Zhang H B 2003 Quasinormal modes prefer supersymmetry? Preprint gr-qc/0309018

[25] Hod S 1998 Bohr's correspondence principle and the area spectrum of quantum black holes Phys. Rev. Lett. 81 4293 (Preprint gr-qc/9812002)

[26] Bekenstein J D 1974 The quantum mass spectrum of the Kerr black hole Lett. Nuovo Cimento 11467

[27] Wheeler J A 1955 Geons Phys. Rev. 97511

[28] Zerilli F J 1970 Gravitational field of a particle falling in a Schwarzschild geometry analyzed in tensor harmonics Phys. Rev. D 22141

[29] Liu H and Mashoon B 1996 On the spectrum of oscillations of Schwarzschild black holes Class. Quantum Grav. 13233

[30] Andersson N 1997 Evolving test-fields in a black hole geometry Phys. Rev. D 55468 (Preprint gr-qc/9607064)

[31] Visser M 1995 Lorentzian Wormholes: From Einstein to Hawking (Woodbury: AIP)

[32] Morris M S and Thorne K S 1988 Wormholes in space-time and their use for interstellar travel: a tool for teaching general relativity Am. J. Phys. 56395

[33] Suneeta V 2003 Quasinormal modes for the SdS black hole: an analytical approximation scheme Phys. Rev. D 68024020 (Preprint gr-qc/0303114)

[34] Andersson N and Onozawa H 1996 Quasinormal modes of nearly extreme Reissner-Nordstrom black holes Phys. Rev. D 547470 (Preprint gr-qc/9607054) 